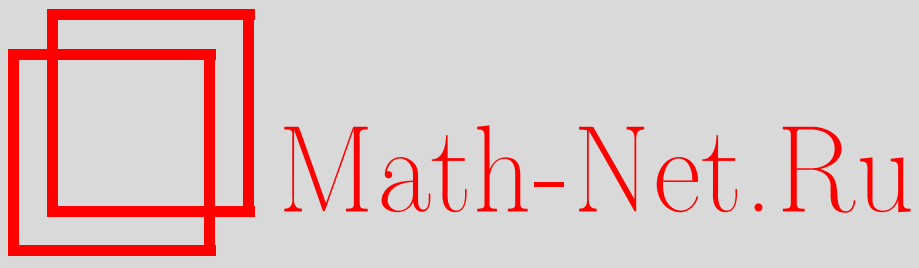

Л. В. Степанова, Н. А. Хомутских, Собственные значения в задаче о трещине антиплоского сдвига в материале со степенным определяющим законом, Вестн. Сам. гос. техн. ун-та. Сер. Физ.-мат. науки, 2006, выпуск 43, 124-131

DOI: https://doi.org/10.14498/vsgtu462

Использование Общероссийского математического портала Math-Net.Ru подразумевает, что вы прочитали и согласны с пользовательским соглашением

http://www.mathnet.ru/rus/agreement

Параметры загрузки:

IP: 3.89 .185 .249

26 апреля 2023 г., 18:37:42

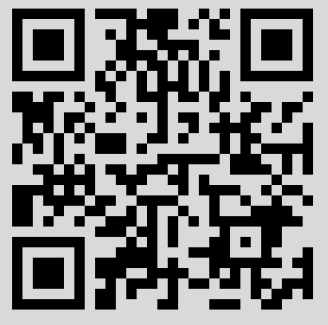




\section{СОБСТВЕННЫЕ ЗНАЧЕНИЯ В ЗАДАЧЕ О ТРЕЩИНЕ АНТИПЛОСКОГО СДВИГА В МАТЕРИАЛЕ СО СТЕПЕННЫМ ОПРЕДЕЛЯЮЩИМ ЗАКОНОМ}

Приведено исследование собственных значений в задаче о трещине антиплоского сдвига в материале со степенным определяющим законом. Дано обобщение метода годографа для определения всего спектра собственных значений задачи. Показано, что собственные значения могут быть найдены также с помощью численного анализа и аналитически с помощьью метода возмущений. Установлено, что все три метода приводят к одинаковым результатам. Приведены некоторые собственные числа для различных значений показателя степени определяющего закона.

В последнее время в современной механике разрушения особый интерес вызывает проблема исследования собственных значений в классической задаче нелинейной механики разрушения - задаче Хатчинсона-Райса-Розенгрена (HRR) [1-6]. Речь идет об исследовании напряженно-деформированного состояния в окрестности вершины трещины в материале со степенным определяющим законом. Одним из широко распространенных подходов исследования является метод разложения по собственным функциям [7], приводящий к обыкновенным дифференциальным уравнениям, и затем - к задаче на собственные значения.

Необходимо отметить, что одно собственное значение, соответствующее задаче HRR $[1,2$, $8-10]$, известно и определяется из дополнительного условия физического характера $[8,10]$. Однако сейчас признана необходимость отыскания всего спектра собственных чисел задачи, и этой проблеме посвящается большое количество работ [3-6, 11]. К сожалению, до сих пор не найдена аналитическая зависимость собственного значения от показателя степени определяющего уравнения (и, например, от собственного значения, отвечающего линейной задаче) для трещин типов I и II. Имеются лишь отдельные результаты, носящие приближенный характер $[3,4,6]$.

Целью настоящей работы является нахождение всего спектра собственных значений для трещины антиплоского сдвига с помощью разных подходов, а именно: посредством численного анализа, с помощью обобщения метода годографа и с помощью метода возмущений, а также сравнение полученных результатов. Проведенные исследования позволят пролить свет на решения более сложных (с математической точки зрения) задач о трещине нормального отрыва и поперечного сдвига.

1. Постановка задачи о трещине антиплоского сдвига в материале со степенным определяющим законом. В случае антиплоского сдвига перемещения происходят только в направлении, перпендикулярном плоскости, содержащей трещину. Предположим, что $u_{1}=u_{2}=0$ и $u_{3}=u_{3}\left(x_{1}, x_{2}\right)$. Тогда только касательные напряжения $\tau_{31}, \tau_{32}$ и сдвиговые деформации

$$
\gamma_{31}=\frac{1}{2} \frac{\partial u_{3}}{\partial x_{1}}, \quad \gamma_{32}=\frac{1}{2} \frac{\partial u_{3}}{\partial x_{2}}
$$

будут отличны от нуля. Условие совместности деформаций имеет вид

$$
\frac{\partial \gamma_{31}}{\partial x_{2}}-\frac{\partial \gamma_{32}}{\partial x_{1}}=0
$$

В данном случае должно удовлетворяться единственное уравнение равновесия

$$
\frac{\partial \tau_{31}}{\partial x_{1}}+\frac{\partial \tau_{32}}{\partial x_{2}}=0
$$

Предполагается, что соотношение между интенсивностью касательных напряжений $\tau$ и интенсивностью деформаций сдвига $\gamma$, где

$$
\tau=\left(\tau_{31}^{2}+\tau_{32}^{2}\right)^{1 / 2} ; \quad \gamma=\left(\gamma_{31}^{2}+\gamma_{32}^{2}\right)^{1 / 2}
$$

остается линейным вплоть до достижения предела текучести $\tau_{0}: \gamma(\tau)=\gamma_{0}\left(\tau / \tau_{0}\right) \quad\left(\tau \leq \tau_{0}\right)$, а затем представляется изотропным нелинейным соотношением

$$
\gamma(\tau)=\gamma_{0}\left(\tau / \tau_{0}\right)^{n} \quad\left(\tau>\tau_{0}\right),
$$

в индексной записи 


$$
\frac{\gamma_{i}}{\gamma_{0}}=\left(\frac{\tau}{\tau_{0}}\right)^{n-1} \frac{\tau_{i}}{\tau_{0}}
$$

или соответственно $\tau(\gamma)=\tau_{0}\left(\gamma / \gamma_{0}\right)^{1 / n} \quad\left(\gamma>\gamma_{0}\right)$. Значение $n=\infty$ отвечает идеальной пластичности, а $n=1-$ идеальной упругости.

Добавив к уравнениям (1) и (2) соотношение (3) между напряжением и деформацией, в общем случае получим нелинейные уравнения, которые должны быть решены с учетом соответствующих граничных условий, а именно, условия отсутствия поверхностных усилий на берегах трещины

и условия симметрии на продолжении трещины

$$
\tau_{32}=0, \quad x_{2}=0, \quad x_{1}<0
$$

$$
\tau_{31}=0, \quad x_{2}=0, \quad x_{1}>0 .
$$

2. Метод разложения по собственным функциям. Постановка задачи. В полярных координатах $(r, \theta)$ с полюсом в вершине трещины уравнение равновесия и условие совместности соответственно имеют вид

$$
\begin{gathered}
\frac{\partial}{\partial r}\left(r \tau_{r z}\right)+\frac{\partial \tau_{\theta z}}{\partial \theta}=0 ; \\
\frac{\partial}{\partial r}\left(r \gamma_{\theta z}\right)=\frac{\partial \gamma_{r z}}{\partial \theta} .
\end{gathered}
$$

Введем скалярную потенциальную функцию

$$
\Phi(r, \theta)=r^{\omega} f(\theta)
$$

такую, что

$$
\tau_{r z}(r, \theta)=\frac{1}{r} \frac{\partial \Phi}{\partial \theta}=r^{\omega-1} f^{\prime}(\theta) ; \quad \tau_{\theta z}(r, \theta)=-\frac{\partial \Phi}{\partial r}=-\omega r^{\omega-1} f(\theta) .
$$

Тогда уравнение равновесия (4) выполняется тождественно, а условие совместности (5) постулирует единственное нелинейное уравнение для единственной неизвестной функции $\Phi(r, \theta)$ :

$$
\frac{\partial}{\partial r}\left\{-r\left[\left(\frac{1}{r} \frac{\partial \Phi}{\partial \theta}\right)^{2}+\left(\frac{\partial \Phi}{\partial r}\right)^{2}\right]^{(n-1) / 2} \frac{\partial \Phi}{\partial r}\right\}=\frac{\partial}{\partial \theta}\left\{\left[\left(\frac{1}{r} \frac{\partial \Phi}{\partial \theta}\right)^{2}+\left(\frac{\partial \Phi}{\partial r}\right)^{2}\right]^{(n-1) / 2} \frac{1}{r} \frac{\partial \Phi}{\partial \theta}\right\} .
$$

Поскольку компоненты тензора деформаций согласно степенному определяющему закону (3) имеют вид

$$
\gamma_{i j}=\frac{3}{2} \frac{f(\tau)}{\tau} \tau_{i j} ; \quad f(\tau)=B \tau^{n}
$$

где $\tau=\sqrt{\tau_{r z}^{2}+\tau_{\theta z}^{2}}=r^{\omega} \sqrt{\left(f^{\prime}\right)^{2}+\omega^{2} f^{2}}$, получаем для $\gamma_{r z}$ и $\gamma_{\theta z}$ следующие выражения компонент тензора деформаций:

$$
\gamma_{r z}=\frac{3}{2} B r^{(\omega-1) n}\left[\left(f^{\prime}\right)^{2}+\omega^{2} f^{2}\right]^{\frac{n-1}{2}} f^{\prime} ; \gamma_{\theta z}=\frac{3}{2} B r^{(\omega-1) n}\left[\left(f^{\prime}\right)^{2}+\omega^{2} f^{2}\right]^{\frac{n-1}{2}}(-\omega f) .
$$

Тогда уравнение (8) принимает вид

$$
f^{\prime \prime}=\frac{-\omega[(\omega-1) n+1] f\left(f^{\prime 2}+\omega^{2} f^{2}\right)-\omega^{2}(n-1) f f^{\prime 2}}{\left(n f^{\prime 2}+\omega^{2} f^{2}\right)} .
$$

Граничные условия на берегах трещины есть условия отсутствия поверхностных усилий и следующие условия симметрии на продолжении трещины:

$$
\begin{array}{lll}
\text { при } \theta=\pi & \tau_{\theta z}=0 & \Rightarrow f(\pi)=0, \\
\text { при } \theta=0 & \tau_{r z}=0 & \Rightarrow f^{\prime}(0)=0,
\end{array}
$$

Таким образом, сформулирована двухточечная краевая задача (9)-(11) для функции $f(\theta)$.

Численный анализ задачи на собственные значения. Для отыскания численного решения перейдем к начальной задаче. Для этого в силу однородности обыкновенного нелинейного дифференциального уравнения (9) можно поставить условие нормировки $f(0)=1$. Таким образом, получена задача на собственные значения: необходимо найти нетривиальное решение 
дифференциального уравнения (9) путем определения собственного значения $\omega$, при котором искомая функция $f(\theta)$ удовлетворяет граничному условию (10) на верхнем берегу трещины.

Численно решая задачу Коши для уравнения (9) методом Рунге-Кугта-Фельберга, при помощи метода пристрелки определяем для различных $n$ другие собственные значения (как ме́ньшие, чем $n /(n+1)$, так и бо́льшие). В рассматриваемом случае метод пристрелки является однопараметрическим: необходимо найти собственное число $\omega$, контролируя выполнение граничного условия (10). В ходе численного счета считалось, что данное граничное условие выполнено, если $|f(\pi)| \leq 10^{-6}$.

Результаты численного анализа приведены в таблице. Угловые распределения компонент тензора напряжений и интенсивности напряжений для различных значений показателя нелинейности и для различных собственных значений показаны на рис. 1 и 2.

Результаты численного анализа собственных чисел

\begin{tabular}{|l|l|l|l|l|l|}
\hline № & \multicolumn{1}{|c|}{$\omega_{-2}$} & \multicolumn{1}{c|}{$\omega_{-1}$} & \multicolumn{1}{c|}{$\omega_{\text {HRR }}=n /(n+1)$} & \multicolumn{1}{c|}{$\omega_{1}$} & $\omega_{2}$ \\
\hline 1 & $-1,5$ & $-0,5$ & 0,5 & 1,5 & 2,5 \\
\hline 2 & $-0,941317015$ & $-0,23029115$ & 0,666666 & 1,360750117 & 2,099539015 \\
\hline 3 & $-0,792823182$ & $-0,183012702$ & 0,75 & 1,303812609 & 1,947821963 \\
\hline 4 & $-0,726928621$ & $-0,164790209$ & 0,8 & 1,271395576 & 1,865587005 \\
\hline 5 & $-0,690099662$ & $-0,155234318$ & 0,833333 & 1,25 & 1,813321658 \\
\hline 6 & $-0,6666666$ & $-0,149366682$ & 0,857142 & 1,23462682 & 1,776918956 \\
\hline 7 & $-0,650471549$ & $-0,145401687$ & 0,875 & 1,222951453 & 1,75 \\
\hline 8 & $-0,638619155$ & $-0,142544449$ & 0,888888 & 1,213730936 & 1,729232382 \\
\hline 9 & $-0,629573341$ & $-0,140388204$ & 0,9 & 1,206234136 & 1,712695266 \\
\hline 10 & $-0,622444719$ & $-0,138703423$ & 0,90909 & 1,2 & 1,699199179 \\
\hline
\end{tabular}

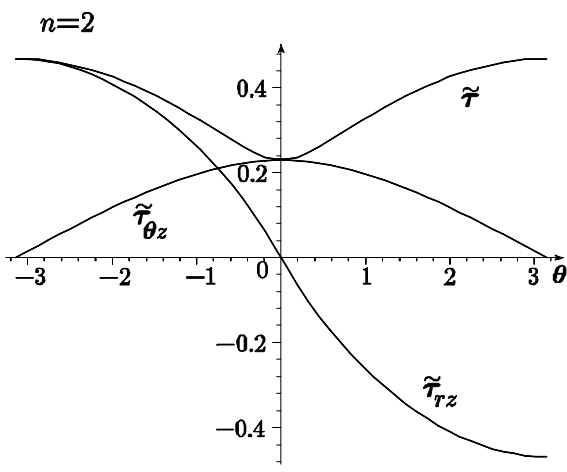

$a$
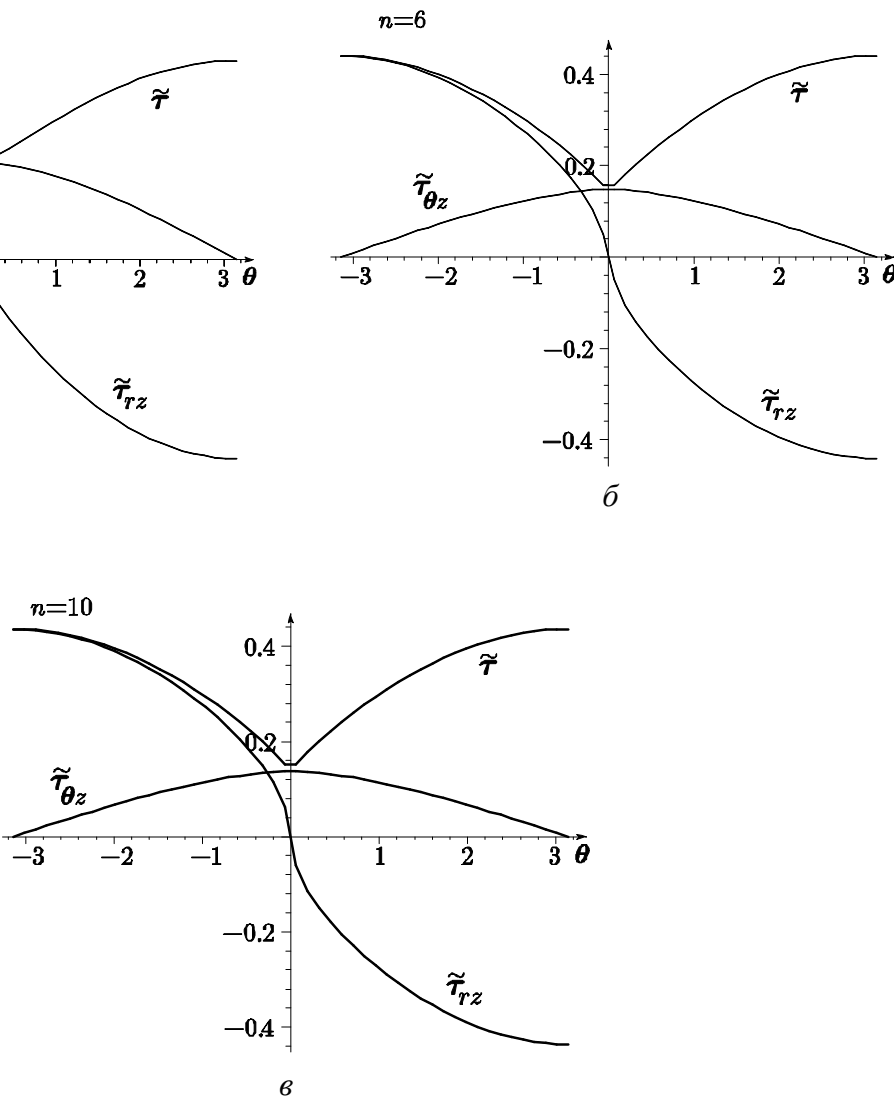

Р и с. 1. Угловые распределения компонент тензора напряжений $9 \%$ напряжений 2 для $\omega_{0}=-1 / 2: a-n=2 ; \sigma-n=6 ; в-n=10$ 

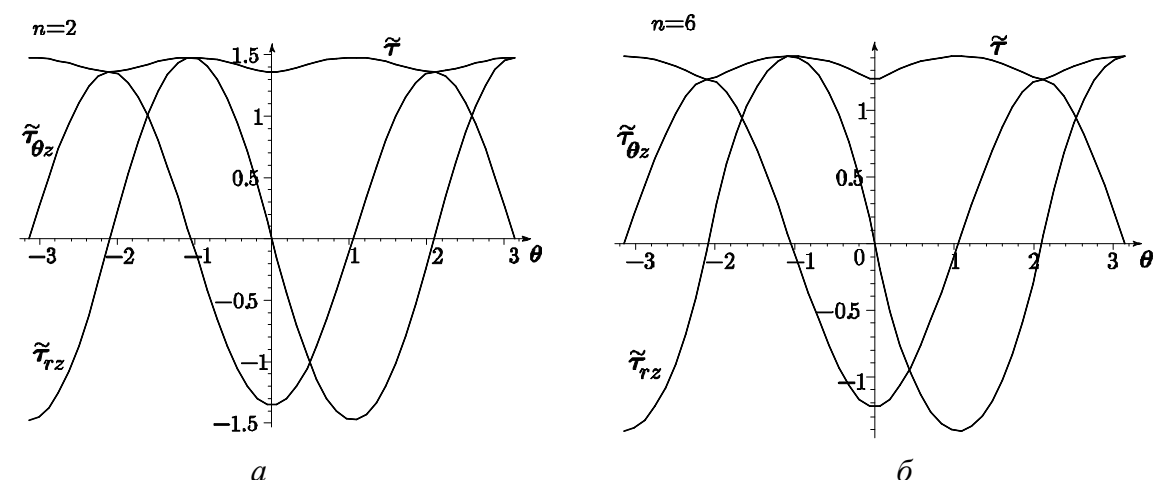

$a$

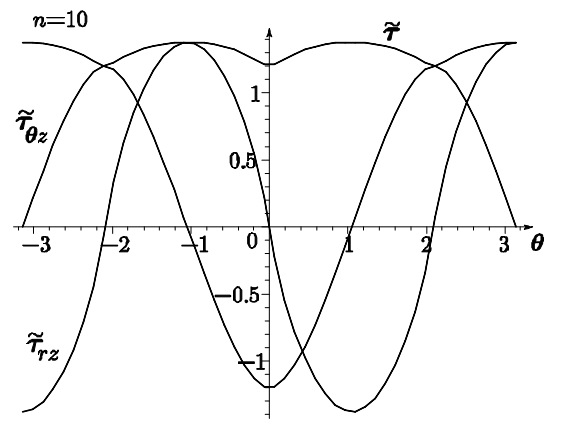

B

Р и с. 2. Угловые распределения компонент тензора напряжений $\mathscr{\%}, \mathscr{Q} / z$ и интенсивности напряжений

3. Обобщение метода годографа. Классическая процедура метода годографа НейбераРайса. Решение Хатчинсона-Райса-Розенгрена. Нелинейные уравнения задач антиплоского сдвига приводятся к линейным уравнениям при переходе к плоскости годографа [7-9], при котором физические координаты рассматриваются как функции компонент тензора напряжений $x_{1}=x_{1}\left(\tau_{31}, \tau_{32}\right), x_{2}=x_{2}\left(\tau_{31}, \tau_{32}\right)$, или, что эквивалентно, как функции компонент тензора деформаций $x_{1}=x_{1}\left(\gamma_{31}, \gamma_{32}\right), x_{2}=x_{2}\left(\gamma_{31}, \gamma_{32}\right)$.

Подобные преобразования приводят уравнение равновесия и условие совместности к виду соответственно.

$$
\frac{\partial x_{1}}{\partial \tau_{31}}+\frac{\partial x_{2}}{\partial \tau_{32}}=0, \quad \frac{\partial x_{1}}{\partial \gamma_{32}}-\frac{\partial x_{2}}{\partial \gamma_{31}}=0
$$

Условие совместности будет выполнено, если ввести функцию компонент тензора деформации $\Phi\left(\gamma_{31}, \gamma_{32}\right)$ такую, что

$$
x_{1}=\frac{\partial \Phi}{\partial \gamma_{31}}, \quad x_{2}=\frac{\partial \Phi}{\partial \gamma_{32}}
$$

Теперь необходимо совершить переход в уравнении равновесия от переменных $\tau_{31}, \tau_{32}$ к независимым переменным $\gamma_{31}, \gamma_{32}$. Используя определяющие соотношения

$$
\gamma_{31}=\frac{\gamma}{\tau(\gamma)} \tau_{31}, \quad \gamma_{32}=\frac{\gamma}{\tau(\gamma)} \tau_{32}
$$

и соотношения (13), можно получить для $\Phi$ линейное дифференциальное уравнение с переменными коэффициентами. Это уравнение имеет наиболее простой вид в полярных координатах $\gamma, \varphi$ на плоскости годографа:

$$
\gamma e^{i \varphi}=\gamma_{32}-i \gamma_{31}, \quad \tau e^{i \varphi}=\tau_{32}-i \tau_{31},
$$

где $\gamma$ - интенсивность деформаций сдвига, $\varphi-$ угол между направлением $\gamma$ и осью $\gamma_{32}$.

В полярной системе координат соотношения (13) представляются в виде

$$
x_{1}=-\sin \varphi \frac{\partial \Phi}{\partial \gamma}-\frac{\cos \varphi}{\gamma} \frac{\partial \Phi}{\partial \varphi}, \quad x_{2}=\cos \varphi \frac{\partial \Phi}{\partial \gamma}-\frac{\sin \varphi}{\gamma} \frac{\partial \Phi}{\partial \varphi} .
$$


Уравнение равновесия в полярной системе координат на плоскости годографа удовлетворяется, если

$$
\frac{\tau(\gamma)}{\gamma \tau^{\prime}(\gamma)} \frac{\partial^{2} \Phi}{\partial \gamma^{2}}+\frac{1}{\gamma} \frac{\partial \Phi}{\partial \gamma}+\frac{1}{\gamma^{2}} \frac{\partial^{2} \Phi}{\partial \varphi^{2}}=0
$$

где $\tau^{\prime}(\gamma)=d \tau / d \gamma$.

Таким образом, в плоскости годографа уравнения задачи становятся линейными. Если оказывается возможным переформулировать физические граничные условия на плоскость годографа, то решение можно будет получить аналитически.

Для того, чтобы получить аналитическую зависимость $\omega=\omega(n)$ для собственных чисел, представим решение уравнения (15) в виде

$$
\Phi(\gamma, \varphi)=\gamma^{\delta} \mathrm{Z}(\varphi)
$$

Подставим (16) в (15), полагая, что $\tau(\gamma)=\gamma^{1 / n}$. Для материала со степенным определяющим законом уравнение (15) в результате сводится к следующему дифференциальному уравнению с постоянными коэффициентами:

$$
\mathrm{Z}^{\prime \prime}+\lambda^{2} \mathrm{Z}=0
$$

где $\lambda^{2}=n \delta(\delta-1)+\delta$.

Классическая процедура метода годографа осуществляет отображение поля деформаций для полубесконечной трещины на положительную полуплоскость деформации, когда угол $\varphi$ на плоскости годографа изменяется в пределах: $-\pi / 2 \leq \varphi \leq \pi / 2$. В решении уравнения (17) $\mathrm{Z}(\varphi)=A \sin (\lambda \varphi)$ величина $\lambda$ определяется из граничных условий:

$$
\frac{\partial Z}{\partial \varphi}=0, \quad \varphi= \pm \frac{\pi}{2} ; Z=0, \quad \varphi=0 .
$$

В этом случае $\lambda_{k}=1+2 k, \quad k=0, \pm 1, \pm 2, \mathrm{~K}$ Следовательно,

$$
\delta_{k}=\frac{(n-1) \pm \sqrt{(n-1)^{2}+4 n \lambda_{k}^{2}}}{2 n} .
$$

Имеем $\Phi(\gamma, \varphi)=\sum_{-\infty}^{\infty} \gamma^{\delta_{k}} Z_{k}(\varphi)$. Согласно (14) $r: \gamma^{\delta_{k}-1}$, следовательно, $\gamma: r^{1 /\left(\delta_{k}-1\right)}$ и

$$
\tau: \gamma^{1 / n}: r^{\frac{1}{n\left(\delta_{k}-1\right)}} .
$$

Таким образом, учитывая (7), получаем искомую аналитическую зависимость для $\omega$ :

$$
\omega_{k}-1=\frac{1}{n\left(\delta_{k}-1\right)}
$$

или

$$
\omega_{k}=1+\frac{2}{-n-1 \pm \sqrt{(n-1)^{2}+4 n \lambda_{k}^{2}}} .
$$

Обобщение метода годографа. Чтобы найти другие собственные числа, отличные от собственного числа $\omega=n /(n+1)$, соответствующего решению HRR, отобразим физическую плоскость на область (плоскости годографа), определяемую неравенствами

$$
-\frac{3}{2} \pi \leq \varphi \leq \frac{3}{2} \pi
$$

Тогда для уравнения (15) изменяются граничные условия на образах берегов трещины:

$$
\begin{gathered}
\frac{\partial \Phi}{\partial \varphi}=0 \text { при } \varphi= \pm \frac{3 \pi}{2} . \\
\text { Исследуя краевую задачу } Z^{\prime \prime}+\lambda^{2} Z=0, \frac{\partial Z}{\partial \varphi}=0 \text { при } \varphi= \pm \frac{3 \pi}{2}, \text { можно найти } Z_{m}(\varphi)= \\
=A_{m} \sin \left(\lambda_{m} \varphi\right) \text { и } \lambda_{m}=\frac{1}{3}+\frac{2}{3} m, \quad m=0, \pm 1, \pm 2, \mathrm{~K},
\end{gathered}
$$




$$
\delta_{1,2}=\frac{(n-1) \pm \sqrt{(n-1)^{2}+4 n\left(\frac{1}{3}+\frac{2}{3} m\right)^{2}}}{2 n}, m=0, \pm 1, \pm 2, \mathrm{~K} .
$$

Для асимптотики дальнего поля напряжений, когда $r \rightarrow \infty$, а $\gamma \rightarrow 0$, необходимо, чтобы в искомом асимптотическом разложении (16):

$$
\Phi(\gamma, \varphi)=\sum_{-\infty}^{\infty} \gamma^{\delta_{k}} Z_{k}(\varphi)=\gamma^{\delta_{1}} Z_{1}+\gamma^{\delta_{2}} Z_{2}+K
$$

каждый следующий член разложения был более высокого порядка малости, чем предыдущий. Согласно (7) $\tau: r^{\omega-1}$, a $\gamma: r^{(\omega-1) n}$. С учетом (20) имеем

$$
\gamma: r^{1 /\left(\delta_{m}-1\right)} \text {. }
$$

Следовательно, для $\gamma \rightarrow 0$ необходимо, чтобы $\delta_{m+1}>\delta_{m}>\delta_{m-1}$. Поэтому выберем собственные значения $\delta_{m}$, удовлетворяющие этому условию, что соответствует знаку «+» в выражении (22) и асимптотике дальнего поля напряжений $(r \rightarrow \infty)$ :

$$
\delta_{m}=\frac{(n-1)+\sqrt{(n-1)^{2}+4 n\left(\frac{1}{3}+\frac{2}{3} m\right)^{2}}}{2 n}, m=0, \pm 1, \pm 2, \mathrm{~K} .
$$

Если отобразим физическую плоскость на область $-5 \pi / 2 \leq \varphi \leq 5 \pi / 2$ плоскости годографа, получим аналогично

$$
\lambda_{m}=\frac{3}{5}+\frac{2}{5} m, \quad m=0, \pm 1, \pm 2, \mathrm{~K} .
$$

Таким образом, собственные значения на плоскости годографа $\delta_{i}$ и $\delta_{-i}(i=0,1,2, \mathrm{~K})$ определяются равенствами:

$$
\begin{aligned}
& \delta_{0}=\delta_{H R R}=\frac{(n-1)-\sqrt{(n-1)^{2}+4 n}}{2 n}=-\frac{1}{n}, \quad \delta_{1}=\frac{(n-1)+\sqrt{(n-1)^{2}+36 n}}{2 n} ; \\
& \delta_{-1}=\frac{(n-1)+\sqrt{(n-1)^{2}+4 n / 9}}{2 n}, \quad \delta_{2}=\frac{(n-1)+\sqrt{(n-1)^{2}+4 n(25 / 9)}}{2 n} ; \\
& \delta_{-2}=\frac{(n-1)+\sqrt{(n-1)^{2}+4 n(9 / 25)}}{2 n}, \quad \delta_{3}=\frac{(n-1)+\sqrt{(n-1)^{2}+4 n(49 / 25)}}{2 n} ; \\
& \delta_{-3}=\frac{(n-1)+\sqrt{(n-1)^{2}+4 n(25 / 49)}}{2 n},
\end{aligned}
$$

Возвращаясь на физическую плоскость, согласно (20) получаем выражения для вычисления собственных чисел $\omega_{i}$ и $\omega_{-i}(i=0,1,2, \mathrm{~K})$ соответственно:

$$
\begin{aligned}
& \omega_{H R R}=1+\frac{2}{-n-1-\sqrt{(n-1)^{2}+4 n}}=\frac{n}{n+1}, \quad \omega_{1}=1+\frac{2}{-n-1+\sqrt{(n-1)^{2}+36 n}} ; \\
& \omega_{-1}=1+\frac{2}{-n-1+\sqrt{(n-1)^{2}+4 n / 9}}, \quad \omega_{2}=1+\frac{2}{-n-1+\sqrt{(n-1)^{2}+4 n(25 / 9)}} ; \\
& \omega_{-2}=1+\frac{2}{-n-1+\sqrt{(n-1)^{2}+4 n(9 / 25)}}, \omega_{3}=1+\frac{2}{-n-1+\sqrt{(n-1)^{2}+4 n(49 / 25)}} ; \\
& \omega_{-3}=\mathrm{K}, \quad \omega_{4}=\mathrm{K} .
\end{aligned}
$$

Выбирая различные значения $n$, можно увидеть, что собственные числа совпадают с найденными ранее в ходе численного анализа.

4. Метод возмущений. Метод возмущений также дает возможность аналитического определения собственного числа $\omega$ как функции показателя степенного определяющего закона $n$ и собственного числа $\omega_{0}$, отвечающего линейной задаче $(n=1)$. Впервые данный подход для 
исследования собственных чисел в задаче о трещине антиплоского сдвига был применен в [11]. Его преимущество состоит в том, что он применим к более сложным (чем случай антиплоского сдвига) задачам.

Для того чтобы найти аналитическое выражение для собственных чисел нелинейного обыкновенного дифференциального уравнения (9), представим собственное число $\omega$ в форме

$$
\omega=\omega_{0}+\varepsilon,
$$

где $\varepsilon-$ отклонение собственного значения $\omega$ для произвольного $n$ от собственного значения $\omega_{0}$, отвечающего линейной задаче. Представим показатель упрочнения $n$ и функцию $f(\theta)$, входящую в выражение (6), в виде

$$
\begin{gathered}
n\left(\varepsilon, \omega_{0}\right)=1+\varepsilon n_{1}\left(\omega_{0}\right)+\varepsilon^{2} n_{2}\left(\omega_{0}\right)+\mathrm{K}+\varepsilon^{k} n_{k}\left(\omega_{0}\right)+\mathrm{K}, \\
f(\varepsilon, \theta)=f_{0}(\theta)+\varepsilon f_{1}(\theta)+\varepsilon^{2} f_{2}(\theta)+\mathrm{K},
\end{gathered}
$$

где $n_{0}$ и $f_{0}(\theta)$ относятся к линейной задаче. Подставим (26), (27) и (28) в уравнение (9), удерживая в асимптотических разложениях $n\left(\varepsilon, \omega_{0}\right)$ и $f(\theta)$ слагаемые вплоть до $\varepsilon^{2}$. Собирая коэффициенты при одинаковых степенях $\varepsilon$, можно найти последовательность обыкновенных дифференциальных уравнений относительно функций $f_{0}, f_{1}, f_{2}, \mathrm{~K}$ :

$$
\begin{array}{ll}
\varepsilon^{0}: & f_{0}^{\prime \prime}+\omega_{0}^{2} f_{0}=0 \\
\varepsilon^{1}: & f_{1}^{\prime \prime}+\omega_{0}^{2} f_{1}=-f_{0} \omega_{0}\left(n_{1}\left(\omega_{0}-1\right)+2\right) ; \\
\varepsilon^{2}: & f_{2}^{\prime \prime}+\omega_{0}^{2} f_{2}=-f_{0}\left[\omega_{0}\left(n_{2}\left(\omega_{0}-1\right)+n_{1}\right)-1\right]-2 \omega_{0} n_{1} \frac{f_{0} f_{0}^{\prime 2}}{f_{0}^{\prime 2}+\omega_{0}^{2} f_{0}^{2}} .
\end{array}
$$

Уравнение (29) описывает линейную задачу, решение которой, удовлетворяющее граничным условиям $f_{0}(\theta=0)=0, f_{0}^{\prime}(\theta=\pi)=0$, имеет вид

$$
f_{0}(\theta)=A \cos \left(\omega_{0} \theta\right) .
$$

Используя это решение, можно легко определить решения следующих уравнений, последовательно находя функции $f_{1}, f_{2}, \mathrm{~K}$. Принимая обычное предположение, согласно которому секулярные слагаемые должны исчезать в решении, получаем последовательность условий для определения $n_{1}, n_{2}, \mathrm{~K}$. В результате для случая, когда $\omega_{0} \neq 1 / 2$, асимптотическое разложение (27) для показателя упрочнения принимает вид [11]

$$
n\left(\varepsilon, \omega_{0}\right)=1+\frac{\omega_{*}}{\omega_{0}-\omega_{*}} \sum_{k=1}^{\infty}\left(\frac{-\varepsilon}{\omega_{0}-\omega_{*}}\right)^{k}-\frac{1}{\omega_{0}-1} \sum_{k=1}^{\infty}\left(\frac{-\varepsilon}{\omega_{0}-1}\right)^{k}=\frac{\omega}{\omega-\omega_{*}}-\frac{\omega}{\omega-1},
$$

где $\omega_{*}=\frac{\omega_{0}^{2}}{2 \omega_{0}-1}$.

Разрешая (33) относительно $\omega$, получаем следующую аналитическую зависимость:

$$
\omega_{1,2}=\frac{\omega_{0}^{2}(n+1)+\left(2 \omega_{0}-1\right)(n-1)}{2 n\left(2 \omega_{0}-1\right)} \pm \frac{\left(\omega_{0}-1\right) \sqrt{\omega_{0}^{2}(n+1)^{2}-\left(2 \omega_{0}-1\right)(n-1)^{2}}}{2 n\left(2 \omega_{0}-1\right)} .
$$

Непосредственная проверка корней $\omega_{1}$ и $\omega_{2}$ позволяет заключить, что искомая зависимость $\omega=\omega\left(n, \omega_{0}\right)$ имеет вид:

$$
\omega=\frac{\omega_{0}^{2}(n+1)+\left(2 \omega_{0}-1\right)(n-1)}{2 n\left(2 \omega_{0}-1\right)}+\frac{\left(\omega_{0}-1\right) \sqrt{\omega_{0}^{2}(n+1)^{2}-\left(2 \omega_{0}-1\right)(n-1)^{2}}}{2 n\left(2 \omega_{0}-1\right)} .
$$

Для $\omega_{0}=1 / 2$ асимптотическое разложение показателя упрочнения принимает форму

$$
n=1-\frac{1}{\omega_{0}-1} \sum_{k=1}^{\infty}\left(\frac{-\varepsilon}{\omega_{0}-1}\right)^{k}=-\frac{\omega}{\omega-1},
$$

что эквивалентно равенству $\omega=n /(n+1)$ и соответствует задаче HRR.

Необходимо отметить, что если указать значение $\omega_{0}$, то формула (34) приобретает тот же вид, что и выражение для вычисления $\omega$, полученное при помощи метода годографа. Выбирая 
различные значения $n$, также можно увидеть, что собственные числа совпадают с найденными ранее в ходе численного анализа и приведенными в таблице.

Выводы и обсуждение результатов. Таким образом, показано, что собственные значения в задаче о трещине антиплоского сдвига в материале со степенным определяющим законом могут быть найдены с помощью численного анализа полученной задачи на собственные значения, с помощью обобщенного метода годографа и метода возмущений.

Установлено, что все три метода приводят к одинаковым результатам, однако последние два подхода дают аналитические зависимости, связывающие искомое собственное значение и показатель нелинейности, тогда как численный анализ позволяет отыскать лишь числовые значения (см. таблицу).

Необходимо отметить, что к задачам о трещине типов I и II, по-видимому, применим лишь метод возмущений, поскольку численный анализ приведет к многопараметрическому методу пристрелки, результаты которого не являются надежными и требуют проверки и дополнительного обоснования.

\section{БИБЛИОГРАФИЧЕСКИЙ СПИСОК}

1. Hutchinson J. W. Singular behavior at the end of tensile crack in a hardening material // J. Mech. Phys. Solids, 1968. Vol. 16. - P. 13-31.

2. Rice J. R., Rosengren G. F. Plane strain deformation near a crack-tip in a power-law hardening material // J. Mech. Phys. Solids, 1968. - Vol. 16. - P. 1-12.

3. Filippi S., Ciavarella M., Lazzarin P. An approximate, analytical approach to the 'HRR'-solution for sharp V-notches // Int. J. of Fracture, 2002. - Vol. 117. - P. 269-286.

4. Chen D. H. Ushijima K. Plastic stress singularity near the tip of a V-notch // Int. J. of Fracture, 2000. - Vol. 106. P. 117-134.

5. Hui C. Y., Ruina A. Why K? High order singularities and small scale yielding // Int. J. of Fracture, 1995. — Vol. 72. — P. 97-120.

6. Meng L., Lee S. B. Eigenspectra and orders of singularity at a crack tip for power-law creeping medium // Int. J. of Fracture, 1998. - Vol. 92. - P. 55-70.

7. Астафьев В. И., Радаев Ю. Н., Степанова Л. В. Нелинейная механика разрушения. — Самара: Сам. ун-т, 2001. $-632 \mathrm{c}$

8. Райс Дж. Математические методы в механике разрушения / В кн.: Разрушение. Т.2. Математические основы теории разрушения / Под ред. Либовица. - М.: Мир, 1975. - С. 204-335.

9. Райс Дж. Напряжения, обусловленные острым вырезом в упрочняющемся упруго-пластическом материале при продольном сдвиге. // Труды американского общества инженеров-механиков. Серия Е: Прикладная механика, 1967. — № 2. — С. 32-46.

10. Хеллан К. Введение в механику разрушения. - М.: Мир, 1988. - 364 с.

11. Anheuser M., Gross D. Higher order fields at crack and notch tips in power-law materials under longitudinal shear // Arch. of Appl. Mech., 1994. — No. 64. - P. 509-518. 